University of Nebraska - Lincoln

DigitalCommons@University of Nebraska - Lincoln

CSE Conference and Workshop Papers

Computer Science and Engineering, Department

\title{
Experiences with Dynamic Circuit Creation in a Regional Network Testbed
}

Pragatheeswaran Angu

University of Nebraska-Lincoln, pangu@cse.unl.edu

Byrav Ramamurthy

University of Nebraska-Lincoln, bramamurthy2@unl.edu

Follow this and additional works at: https://digitalcommons.unl.edu/cseconfwork

Part of the Computer Sciences Commons

Angu, Pragatheeswaran and Ramamurthy, Byrav, "Experiences with Dynamic Circuit Creation in a Regional Network Testbed" (2011). CSE Conference and Workshop Papers. 214.

https://digitalcommons.unl.edu/cseconfwork/214

This Article is brought to you for free and open access by the Computer Science and Engineering, Department of at DigitalCommons@University of Nebraska - Lincoln. It has been accepted for inclusion in CSE Conference and Workshop Papers by an authorized administrator of DigitalCommons@University of Nebraska - Lincoln. 


\title{
Experiences with Dynamic Circuit Creation in a Regional Network Testbed
}

\author{
Pragatheeswaran Angu and Byrav Ramamurthy \\ Department of Computer Science and Engineering \\ University of Nebraska-Lincoln \\ Lincoln NE 68588-0115 U.S.A. \\ Email: \{pangu,byrav\}@cse.unl.edu
}

\begin{abstract}
In this paper we share our experiences of enabling dynamic circuit creation in the GpENI network. GpENI is a network research testbed in the mid-west USA involving several educational institutions. University of Nebraska-Lincoln is involved in provisioning dynamic circuits across the GPENI network among its participating universities. We discuss several options investigated for deploying dynamic circuits over the GpENI network as well as our demonstration experiments at the GENI engineering conferences. UNL has also collaborated with ProtoGENI project of University of Utah and Mid-Atlantic Crossroads (MAX) facility of Washington DC to create interdomain dynamic circuits.
\end{abstract}

\section{INTRODUCTION}

Great Plains Environment for Network Innovation (GpENI) [1] [2] is an international programmable testbed in the midwest of United States. The University of Nebraska-Lincoln (UNL), University of Kansas (KU), Kansas State University (KSU) and University of Missouri Kansas City (UMKC) are the participating universities in the midwest along with various other universities in Europe and Asia. UNL is involved in enabling GpENI to provision dynamic circuits among its participating universities. Each GpENI site has a Netgear Ethernet switch which will be used for the creation of dynamic circuits. UNL has also collaborated with ProtoGENI [3] project of University of Utah and Mid-Atlantic Crossroads (MAX) facility of Washington DC to create inter-domain dynamic circuits. DYNES [4] project is a recent and similar effort to create a dynamic circuit testbed in US.

The paper is organized as follows. In Section II we describe the background concepts involved in this paper and in Section III we explain the options for creating dynamic circuit network service in GpENI. In Section IV we describe the options for creating dynamic circuit network service between GpENI and MAX and we explain a demo configuration for transferring large scientific data using Dynamic Circuit Network in Section $\mathrm{V}$ and we conclude the paper in Section VI.

\section{BACKGROUND}

\section{A. Dynamic Circuits}

Dynamic Circuit Network (DCN)/Interoperable On-demand Network (ION) [5] is a networking service in Internet2 that provides researchers the ability to create short-term circuits of large bandwidth across the network. These circuits are created for bandwidth-intensive applications that are run over the Internet 2 backbone network. This service uses both the software components of OSCARS [6] and DRAGON [7] project to create dynamic circuits across various domains and across various network technologies. The circuits are created and deleted using the Web User Interface provided by the OSCARS software components. The Inter Domain Controller (IDC) is basically the entity managing the circuit creation and deletion along with user authentication and authorization mechanisms in an Autonomous System (AS) or local domain. Internet 2 uses the ION service to transfer large scientific data for projects such as Large Hadron Collider (LHC) and Compact Muon Solenoid (CMS).

\section{B. ProtoGENI}

ProtoGENI [3] is a control framework belonging to the NSF/GPO-funded GENI project. ProtoGENI uses the Emulab software and PlanetLab to create a slice of computing as well as networking resource across ProtoGENI testbed.

\section{DRAGON}

Dynamic Resource Allocation via GMPLS Optical Networks (DRAGON) [7] was a NSF funded project to dynamically provision network resources across various domains and across heterogeneous networking technologies. GMPLS [8] is the key protocol used to create circuits spanning across both optical and Ethernet domain and hence DRAGON creates a Layer 1 virtual circuit. A set of software components has been developed to leverage this capability across a testbed over the Washington D.C area. The major components of DRAGON software are VLSR (Virtual Label Switched Router), NARB (Network Aware Resource Broker), ASTB (Application Specific Topology Builder), RCE (Resource Computation Engine). As DRAGON provides the capability to create circuits that span across various domains the NARB acts as the entity that represents a local domain or Autonomous System (AS). In each domain each switch needs to be configured separately for creating a circuit and hence VLSRs acts as the entity controlling the switches. The RCE and ASTB are used for computing the resources required for creating circuits. Hence a particular DRAGON domain will have a NARB and one or more VLSR depending upon the number of switches in the domain. 


\section{OSCARS}

On-Demand Secure Service and Advance Reservation System (OSCARS) is a networking service deployed in the DoE ESnet to create dynamic, deterministic and secure circuits across the ESnet network. MPLS [9] and RSVP [10] are the key protocols used to create advance reservations of bandwidth using the software components developed as part of OSCARS project. The Label Switched Path (LSP)s are created using MPLS both in Layer 2 and Layer 3 using OSCARS software. The circuits are created and deleted using a web interface provided by OSCARS and hence this method is adopted in the DCN/ION project as interface for managing virtual circuits. The major software components of the OSCARS are Reservation Manager (RM), Path Setup Subsystem (PSS) and Bandwidth Scheduler Subsystem (BSS), Authentication, Authorization and Audition Subsystem (AAA). The RM, PSS, BSS are used for reserving resources and creation and deletion of actual circuits in the network and AAA is used to provide authentication mechanisms using X.509 certificates.

\section{E. VLAN}

Virtual LAN is a networking technology used to provide secure and reliable transport between hosts that are not physically connected to each other. IEEE 802.1Q is the most commonly used standard for VLANs and it has been implemented in most of the commercially available switches. VLAN tag is a 32-bit field added to the Ethernet frame, which has a 12bit field called VLAN ID specifying the VLAN number of the packet transmitted over the network. The VLAN number is the entity which differentiates packets of different virtual circuits over a network. There are two approaches to assign VLAN membership, static VLANs and dynamic VLANs. An Ethernet packet carrying a (non-default) VLAN tag is said to be a "tagged" packet and the one carrying default VLAN tag is said to be an "untagged" packet. The static vlans are created by assigning ports to VLAN and dynamic vlans are created using software such as CiscoWorks 2000 in Cisco managed switches and it can also be created using SNMP. VLANs are mostly used by the corporate networks to separate traffic of various applications which share the same network infrastructure. The VLAN tags are added when the packet enters the corporate network and they are removed when they leave the network.

\section{F. $\operatorname{Qin} Q$}

$\mathrm{Q}$ in $\mathrm{Q}$ or Double tagging is a method to add one more outer VLAN tag to already tagged packets. This is used by the Internet Service Providers to separate network traffic between different user groups so that one user group will be isolated from another group. However each member in a group can have their packets tagged differently so that they can protect their packets from other members of the same group. IEEE 802.1ad is the standard specifying this method of double tagging the packets sent over the network. Similar to VLAN tagging the outer tags are added once the packets with inner tag enters the corporate network and the outer tag alone is removed when the packets leave the network.

\section{DCN IN GPENI}

In the following section we first explain the current network infrastructure of the GpENI and the changes needed in the current infrastructure to establish DCN across GpENI.

\section{A. Current GpENI Network Configuration}

GpENI's basic connectivity (see Figure 1) is designed as a single Ethernet broadcast domain capable of transporting arbitrary VLANs. All the four GpENI universities (UNL, KSU, KU and UMKC) are connected to their own interface in GPN Cisco 6509 Ethernet switch (GPN Switch) in Kansas City POP and all these interfaces are configured to the same VLAN number 125 . UNL has direct fiber connection of capacity 1GigE to the GPN switch transported through Ekinops DWDM equipment. UMKC connects to the GPN switch using L2TP tunneling through MOREnet infrastructure. KSU and KU form a single MPLS domain in the KanREN network infrastructure and are connected to the GPN switch through VPLS. The CoreDirector CI switch which connects to Internet2 at the Kansas City PoP is also connected to the GPN switch using a 10GigE link. Each university has a Netgear GSM7224 switch and a node cluster connected to the switch. We have modified the DCN software suite to support the Netgear GSM7224 switch. Hence we can create dynamic circuits between the universities with DCN software running over these switches if the infrastructure in Kansas City PoP supports it. The limitation is that, as these Netgear switches do not have per-VLAN bandwidth policing feature, they do not have the capability to create circuits of specific bandwidth as requested by the user.

\section{B. Option 1: GpENI Network Connectivity with DCN (using GPN switch)}

The GPN switch is a production switch that carries traffic between the four GpENI universities and is also not supported by the current DCN/ION software suite. This option (see Figure 2) analyses the possiblity of using the GPN switch for creating DCN circuits between GpENI universities. There are two ways (1(a) and 1(b)) in which the GPN switch can be configured to enable DCN circuits between GpENI universities. One way (1(a)) is to configure static VLANs over the GPN switch so that DCN circuits can be created between universites only with the pre-configured VLAN tags. Hence this involves creating a table of VLAN tags for all possible source and destination of DCN circuits between GpENI universities and configuring them appropriately in the GPN switch. In this case the IDC and two VLSRs:one for controlling the Ciena CoreDirector CI switch in UNL and another for controlling all the Netgear GSM7224 switches in all the GpENI universities, will be located in the UNL. Hence we will be able to create, delete and modify dynamic circuits over the web interface provided by the DCN/ION software suite between these universities. Another way (1(b)) is to configure Q-in-Q cloud in the GPN 6509 switch with VLAN 125 so that it acts as a pass through for packets of any VLAN tag generated by any of the GpENI universities. Hence in this 


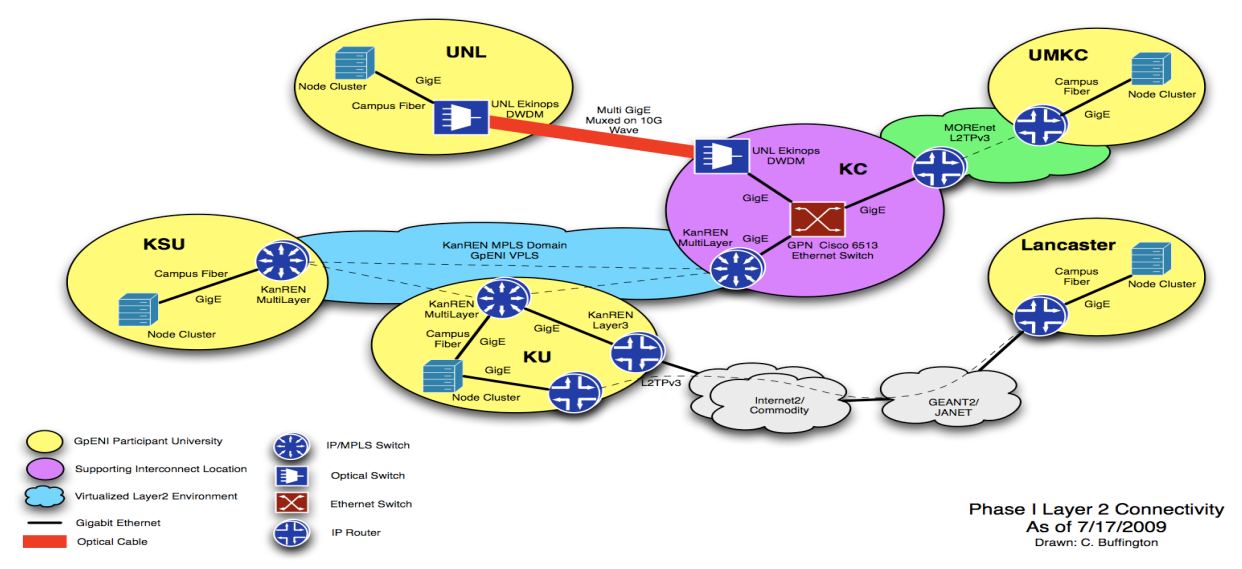

Fig. 1. GpENI Current Network Connectivity.

(source: https://wiki.ittc.ku.edu/gpeni/Image:GpENI-L2.png)

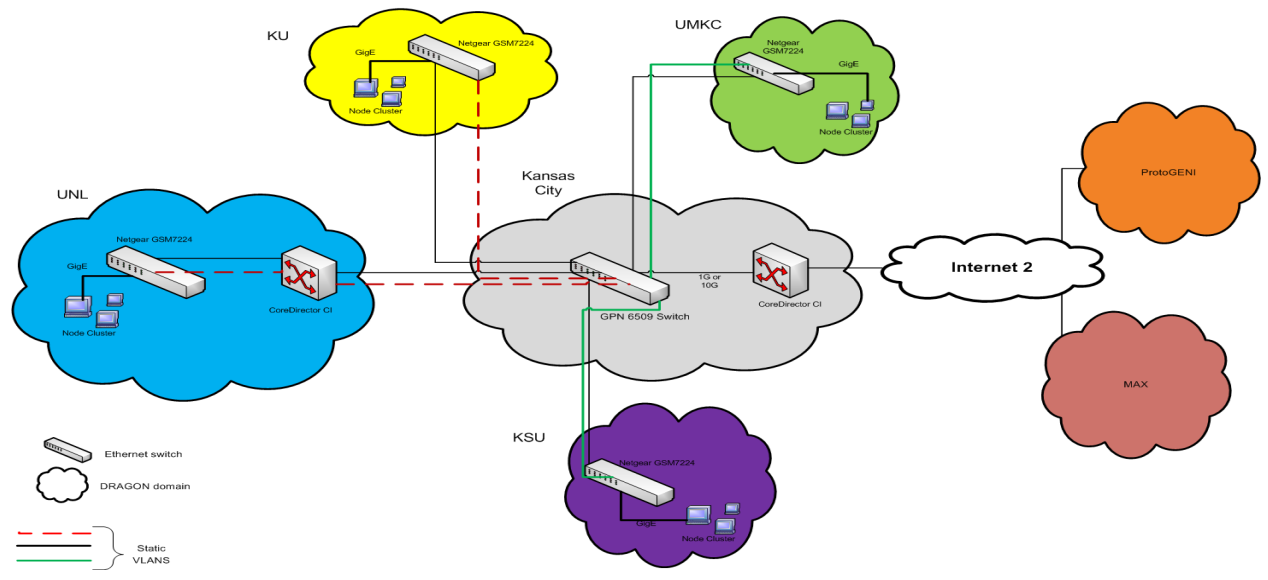

Fig. 2. Option 1: DCN in GpENI (using GPN switch).

case also, the IDC and two VLSRs will be placed at UNL, but the only difference is that we will be able to create circuits of arbitrary VLAN tags between the GpENI universities. Though the advantage of this method over the previous is the freedom of choice of VLAN tags, the drawback is that, because of the Q-in-Q cloud the packet transmitted by any university will be broadcasted to all the 4 GpENI universities.

\section{Option 2: GpENI Network Connectivity with DCN (using GpENI switch)}

This option (see Figure 3) requires acquiring a new Ethernet switch (GpENI switch), which is already supported by the DCN/ION software suite and replacing the existing GPN switch with the GpENI switch. Hence this option requires all the 4 GpENI universities to have a layer 2 connectivity to the GpENI switch and one interface of the GpENI switch will be connected to the GPN switch. The dedicated switch at Kansas City for this option could also be a switch placed by the ProtoGENI group at this location. Funds and approvals are being sought for the deployment of a GpENI switch at Kansas City PoP. The IDC of the DCN/ION can be placed at UNL or Kansas City PoP and two VLSRs: one for controlling the CoreDirector CI and another for controlling the Netgear switches, can be placed in UNL or Kansas City PoP. Hence we will be able to create dynamic circuits of desired bandwidth of arbitrary VLAN tag between any of the GpENI universities with this network infrastructure. The Ciena CoreDirector in Kansas City is shown as connected to the GpENI switch because this will be the Connector for GpENI universities to connect to the Internet2 infrastructure.

\section{Decision}

We have chosen option 1 mentioned in Section III-B after considering the cost factors involved in the option 2. We have shown a demo of this in several GENI confereces GEC7 and GEC8. 


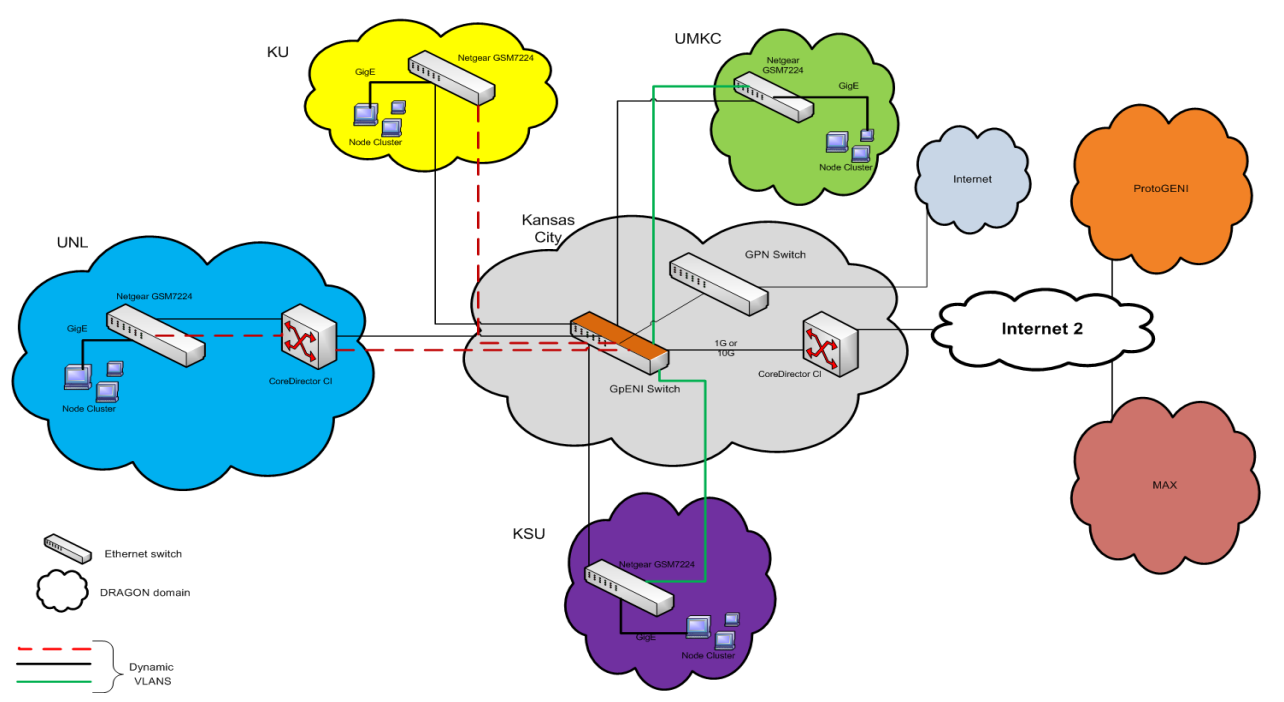

Fig. 3. Option 2: DCN in GpENI (using GpENI switch).

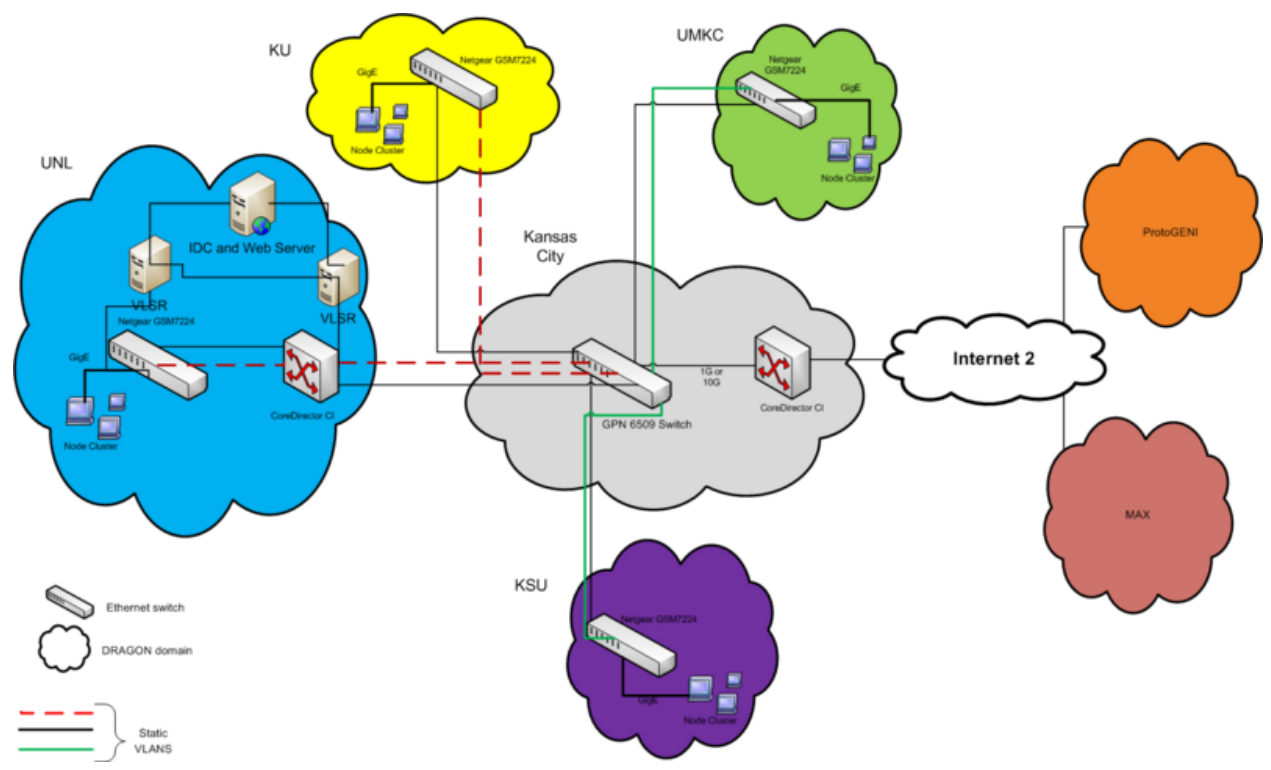

Fig. 4. Connection to MAX using GPN switch and Internet2..

\section{DCN BETWEEN GPENI AND MAX}

\section{A. Option 1: GpENI Network Connectivity with MAX (using GPN switch)}

This option (see Figure 4) discusses using the existing GPN switch for creating DCN network within GpENI. It further discusses the two ways: (a) static VLAN and (b) Qin-Q, to achieve this goal. In the first method, static VLANs are configured in the GPN switch so that DCN circuits can be created between universities with predefined VLAN tags. Hence this involves creating a table of VLAN tags for all possible source and destination of DCN circuits between GpENI universities and configuring them appropriately at the
GPN switch. Hence to connect to MAX in this method we need to configure one more VLAN tag for each university or configure a VLAN tag so that all universities use DCN to MAX only with this VLAN tag. This is basically a choice between individual circuits from each university to MAX or to have a single broadcast domain to MAX. These VLAN tags need to be configured to the interface of GPN in which the CoreDirector of Kansas City PoP is connected to the GPN switch. In this method we need to have an IDC controlling the creation of circuits with the predefined tags from each university. Hence the IDC could be placed in UNL and we need a VLSR for controlling all the Netgear switches of 
all the GpENI universities and a VLSR for controlling the CoreDirector. We can configure one of the PCs to act as a web server and configure the OSCARS software in it so that each university can create and delete circuits using the web interface of OSCARS software.

In the second method, Q-in-Q is configured in the GPN switch so that it acts as a pass through for packets of any VLAN tag generated by any of the GpENI universities. Hence to connect to MAX in this method we need to just include the interface of the GPN switch which is connected to the CoreDirector of the Kansas City PoP in the Q-in-Q cloud. In this manner we can create circuits of any VLAN tag from any of the GpENI university to the MAX. In this also the IDC placement and VLSR placement are similar to the method above but the only difference is that users can create circuits with arbitrary VLAN tag to MAX. In both of the above methods we can also have separate VLSR in each GpENI university creating individual DRAGON domain in each GpENI university. Instead if we have a VLSR for controlling all the Netgear Switches then we have only one DRAGON domain representing all the GpENI universities.

\section{B. Option 2: GpENI Network Connectivity with ProtoGENI (using GPN switch)}

ProtoGENI has a $10 \mathrm{G}$ backbone in the Internet 2 network and it has already deployed its nodes (which includes HP 5400 switches, NetFPGA cards, and 2 PCs) at 3 Internet2 sites (Kansas City, Salt Lake City and Washington D.C.). Currently, in the HP Procurve switch deployed at Kansas City, there are no free 10GigE ports available. Hence, in this option (see Figure 5) we create a $1 \mathrm{GigE}$ connection between the GPN switch at Kansas City and the HP procurve switch in the ProtoGENI node and using this connection to connect to MAX.

Currently in the GpENI network all the universities deliver untagged packets to the GPN switch. Since ProtoGENI requires the packets to be tagged with specific VLAN, the Q-in-Q cloud need to be setup in the GPN switch and each university is required to transmit packets to the GPN switch with predefined outer VLAN tag agreed with ProtoGENI. Qin-Q would be used on the GPN switch to alleviate the need for VLAN number coordination within GPN. The component manager of the ProtoGENI needs to be setup so that we could request dynamic circuits from GpENI to any node of ProtoGENI. In this case we will be using the client software of ProtoGENI instead of DCN to create dynamic virtual circuits. However the circuits can only be created from and to the ProtoGENI nodes and hence the traffic inside GpENI will remain as a broadcast domain.

\section{Option 3: GpENI Network Connectivity with MAX (using GpENI switch)}

This option (see Figure 6) discusses acquiring a new Ethernet switch (GpENI switch), which is already supported by the DCN/ION software suite and replacing the existing GPN switch with the GpENI switch. MAX network can be connected in this method by just connecting the CoreDirector of Kansas City PoP to the GpENI switch. We can have a dedicated IDC controlling the GpENI switch and 1 VLSR controlling all the Netgear switches and a VLSR controlling the CoreDirector in UNL campus. In this case only the dynamic circuit from or to the UNL need to be in the order of $50 \mathrm{Mbps}$ as CoreDirector is in the path. Otherwise the dynamic circuits between other universities could be of any bandwidth capacity supported by the interface of the GpENI switch. Having CoreDirector participate in dynamic circuit is just a choice and hence if we want to create dynamic circuits of any bandwidth to UNL we could remove the VLSR controlling the CoreDirector switch and make it as a pass through switch, simply passing the traffic to other end irrespective of the packets VLAN tag. In this method the IDC needs to be located in the Kansas City PoP and the VLSRs could be in each university or we could have one VLSR controlling all the Netgear Switches located in the UNL. The GpENI switch can be connected to Internet 2 with $\mathrm{DCN}$ or it can be connected to ProtoGENI backbone also if we have a connection between the GpENI switch and the HP Procurve switch of ProtoGENI node.

\section{Decision}

We have chosen both options 1 and 2 and at present we have option 1 (b) configured in the Kansas City PoP. We have shown demos of options $1 \& 2$ in GENI conferences and we will pursue option 3 when more funds are available.

\section{DEMO OF TRANSFERRING CMS DATA WITH DCN}

We have shown a demo of transferring Compact Muon Solenoid (CMS) data using a dynamic circuit established from UNL to MAX in GLOBECOM 2010 conference. In UNL we established a Layer 2 connection between the Prairiefire super computer and the Netgear switch. The GPN switch in the Kansas City PoP was connected to the Internet2's Juniper switch via a static VLAN. We established a dynamic circuit of this VLAN from Internet2 switch to a planetlab node in the MAX domain using MAX IDC. We used UNL's IDC to create circuit of this VLAN id from Netgear to the GPN switch which completes the experimental setup for transferring CMS data to the planetlab node via these dynamic circuits. These configurations enabled the Prariefire node to have a Layer 2 connection to the planetlab node in the MAX and we transferred the CMS data over this circuit. A researcher in the MAX domain or anywhere in Internet2 site can use this service to perform experiments over prarifire node cluster and transfer the result to his place.

\section{CONCLUSION}

The creation of dynamic circuit network enables researchers to transfer large scientific data for short duration of time reliably and quickly without going through the current best effort traffic nature of the Internet. Enabling a regional network for dynamic circuit network needs changes to the configurations in the production switches used to connect participating 


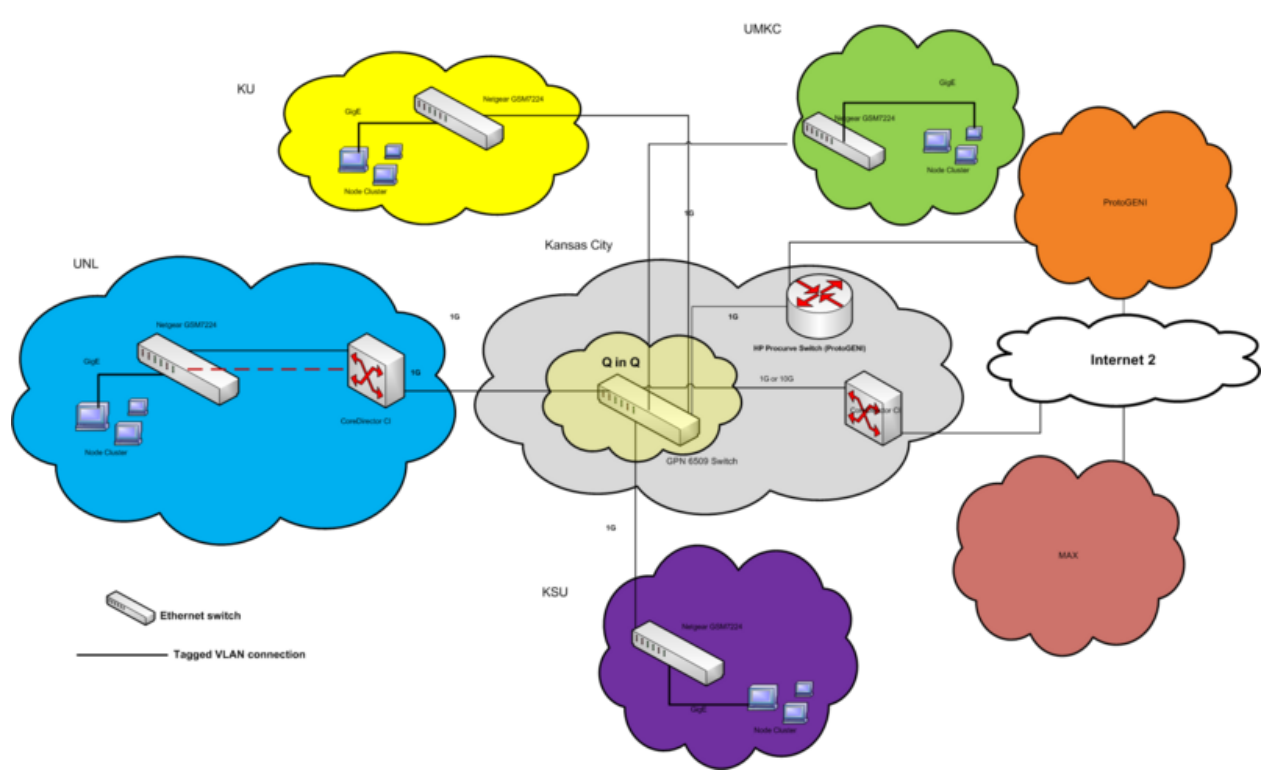

Fig. 5. Connection to MAX using GPN switch,ProtoGENI and Internet2

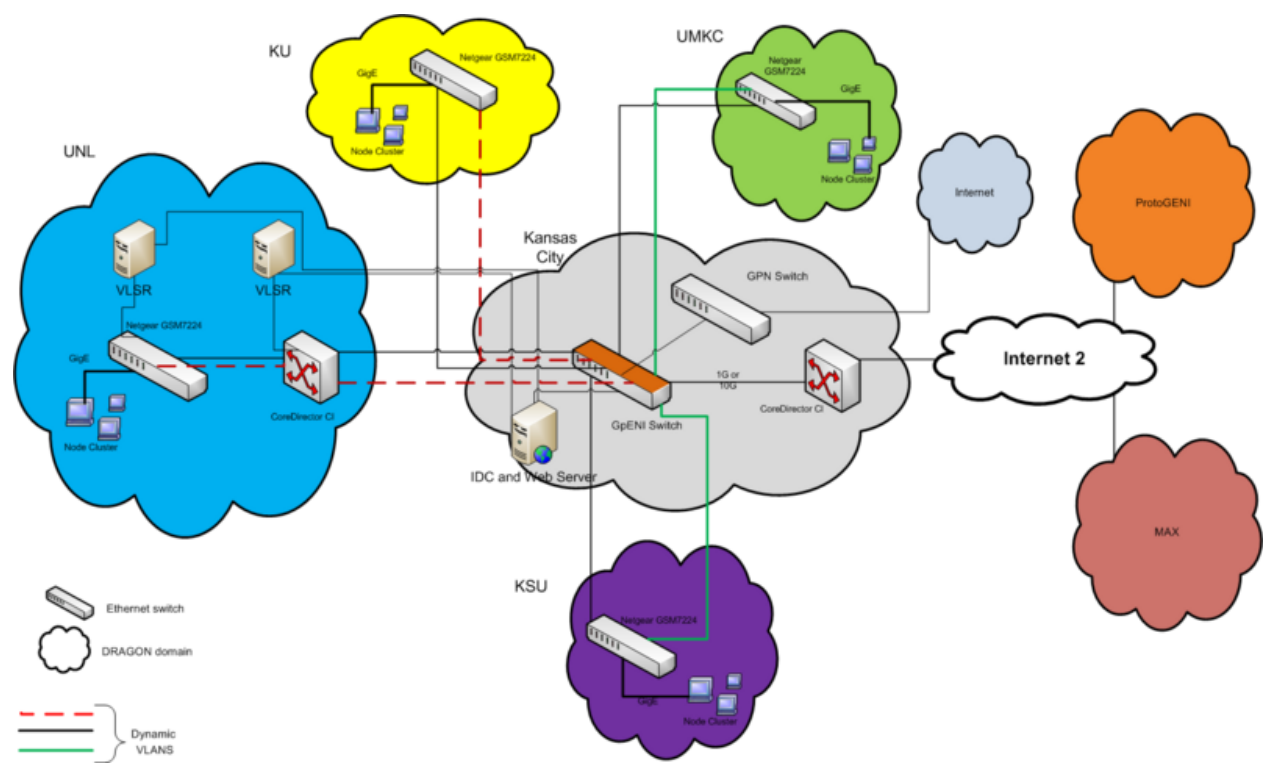

Fig. 6. Connection to MAX using GpENI switch.

institutions as well as designing the control plane and data plane for their network domain.

\section{ACKNOWLEDGMENT}

We thank Kent G Christensen (IS, UNL), Xi Yang (USC/ISI East), Tom Lehman (USC/ISI East), James Sterbenz (University of Kansas), Rob Ricci (ProtoGENI), Cort Buffington (GPN) for their efforts to create the dynamic network service in GpENI. We also thank the GPN operational staff at MORENet. We thank NSF/BBN for its support of this work through a GENI project.

\section{REFERENCES}

[1] http://www.gpeni.net.

[2] J. P. S. e.t al, "The great plains environment for network innovation (gpeni): A programmable testbed for future internet architecture research," pp. 1-14, TRIDENTCOM, 2010.

[3] http://www.protogeni.net/trac/protogeni.

[4] http://www.internet2.edu/ion/dynes.html.

[5] http://www.internet2.edu/ion.

[6] http://www.es.net/oscars/.

[7] http://dragon.maxgigapop.net/twiki/bin/view/DRAGON/Network.

[8] http://www.ietf.org/rfe/rfc3471.txt.

[9] http://www.ietf.org/rfc/rfc3477.txt.

[10] http://www.ietf.org/rfc/rfc2205.txt. 